\title{
Using a Science Gateway to Deliver Sim Vascular Software as a Service for Classroom Instruction
}

\author{
Nathan M. Wilson \\ Open Source Medical Software \\ Corporation \\ Santa Monica, CA \\ nwilson@osmsc.com \\ Marcus A. Christie \\ Science Gateways Research Center, \\ Pervasive Technology Institute, \\ Indiana University \\ machrist@iu.edu
}

\author{
Suresh Marru \\ Science Gateways Research Center, \\ Pervasive Technology Institute, \\ Indiana University \\ smarru@iu.edu
}
Gabriel D. Maher
Stanford University
Stanford, CA
gdmaher@stanford.edu

\author{
Eroma Abeysinghe \\ Science Gateways Research Center, \\ Pervasive Technology Institute, \\ Indiana University \\ eabeysin@iu.edu \\ Adam R. Updegrove \\ University of California, Berkeley \\ Berkeley, CA \\ updega2@berkeley.edu
}

\author{
Marlon Pierce \\ Science Gateways Research Center, \\ Pervasive Technology Institute, \\ Indiana University \\ marpierc@iu.edu
}

\author{
Alison L. Marsden \\ Stanford University \\ Stanford, CA \\ amarsden@stanford.edu
}

\begin{abstract}
SimVascular (http://www.simvascular.org) is open source software enabling users to construct image-based, patient-specific anatomic models and perform realistic blood flow simulation useful in disease research, medical device design, and surgical planning. The software consists of two core executables: a front-end application and a flow solver. The front-end application enables users to create patient-specific anatomic models from imaging data, generate finite-element meshes, prescribe boundary conditions, and set up an analysis. The finite-element based blood flow solver utilizes MPI and is massively scalable. SimVascular has been successfully integrated into graduate level courses on cardiovascular modeling at multiple institutions including Stanford, UC Berkeley, Purdue, and Marquette to introduce state-of-the-art modeling to the students and provide a basis for hands-on projects. While the front-end application can be installed and run on a laptop, the flow solver requires high performance computing (HPC) for realistic problem sizes. This provides a significant challenge for instructors as many students are unfamiliar with HPC, and local resources might be limited or difficult to administer. There is also a need to provide user and group management capabilities for courses: students should authenticate using campus credentials, instructors should be able to access students' work, and students' access to computing allocations should be limited. Our poster will detail an Apache Airavata-based science gateway to address these needs. XSEDE's Comet provides the backend computing power. This approach allows the SimVascular

Permission to make digital or hard copies of part or all of this work for personal or classroom use is granted without fee provided that copies are not made or distributed for profit or commercial advantage and that copies bear this notice and the full citation on the first page. Copyrights for third-party components of this work must be honored.

For all other uses, contact the owner/author(s).

PEARC '18, fuly 22-26, 2018, Pittsburgh, PA, USA

(C) 2018 Copyright held by the owner/author(s)

ACM ISBN 978-1-4503-6446-1/18/07.

https://doi.org/10.1145/3219104.3229242
\end{abstract}

team to provision HPC resources and install and maintain the software providing students access at institutions across the country. The science gateway interface provides access to SimVascular's flow solver, while allowing students to use SimVascular's desktop interfaces.

\section{CCS CONCEPTS}

- Computing methodologies $\rightarrow$ Massively parallel and highperformance simulations; • Applied computing $\rightarrow$ Imaging; - Software and its engineering;

\section{KEYWORDS}

ACM proceedings, Apache Airavata, Science Gateway

ACM Reference Format:

Nathan M. Wilson, Suresh Marru, Eroma Abeysinghe, Marcus A. Christie, Gabriel D. Maher, Adam R. Updegrove, Marlon Pierce, and Alison L. Marsden. 2018. Using a Science Gateway to Deliver SimVascular Software as a Service for Classroom Instruction. In PEARC '18: Practice and Experience in Advanced Research Computing, fuly 22-26, 2018, Pittsburgh, PA, USA. ACM, New York, NY, USA, 4 pages. https://doi.org/10.1145/3219104.3229242

\section{INTRODUCTION}

Hemodynamics is central to the origin, progression, and treatment of cardiovascular disease. Mechanical stimuli such as blood pressure, velocity, wall shear stress, and wall deformation are known to influence cardiovascular disease progression, and also factor into personalized treatment planning decisions. Simulations have recently been applied to treatment planning and risk stratification for numerous diseases including coronary artery disease, bypass graft failure, cardiomyopathy, pulmonary hypertension, aneurysm growth and rupture, and congenital heart disease. Methodological improvements in patient-specific image-based modeling together 
with advances in computational methods have increased simulation accuracy and fidelity. Blood flow simulations using medical image-based anatomical models now offer a non-invasive technique to determine accurate flow velocity, wall shear stress, and other clinically relevant quantities to augment medical imaging. The open-source code SimVascular project has contributed to numerous advances in fundamental disease research, surgical planning, and medical device design. An accompanying publicly available data repository (www.vascularmodel.org) provides patient-specific image data, models and simulation results of over 120 clinical cases from different regions of the vasculature, including cerebrovascular, coronary, aortofemoral, pulmonary and congenital heart disease. In this paper, we summarize the development of the SimVascular Gateway, which provides access to SimVascular software as a service for classroom and educational usage modes. The SimVascular Gateway is developed using Apache Airavata [4] and is hosted on the SciGaP.org platform for science gateways. SimVascular software is delivered to the community through XSEDE resources [6]. Development of the SimVascular Science Gateway and integration with XSEDE is supported by XSEDE's Extended Collaborative Support Services [7].

\section{STEPS FOR IMAGE-BASED, PATIENT-SPECIFIC BLOOD FLOW SIMULATION}

\subsection{Medical Image Visualization}

The process of image-based patient specific blood flow simulation in SimVascular typically involves six major steps (see Fig. 1). First, the analyst will load and visualize a volumetric medical image dataset. Common image data formats supported including the standard Digital Imaging and Communications in Medicine (DICOM) format as well as several formats from popular open-source code packages such as the Visualization Toolkit (VTK, www.vtk.org). This step in the process is highly interactive as the user can use a variety of medical imaging visualization techniques to become familiar with the data. In addition to visually assessing the quality of the image data, an analyst may make decisions regarding the anatomic region of interest to model and the appropriateness of certain boundary conditions for subsequent analysis. Files for a single volumetric image can range from $20 \mathrm{MB}$ to more than $2 \mathrm{~GB}$ so this step is best performed on a high-end laptop or desktop computer with high performance graphics capabilities.

\subsection{Anatomic Model Construction}

During the second stage in the workflow, an analyst will select approximate centerline paths for all vessels of interest in the volume (see Fig. 2). Analysts must weigh the tradeoffs between anatomic model complexity, simulation accuracy, and computational cost. An extensive anatomic model may reduce the impact of boundary conditions and account for more patient-specific anatomic variation, but could significantly increase computational time and complexity of the analysis. The third stage involves image segmentation in 2-D planes perpendicular to the centerline path. For each blood vessel, the analyst performs a series of segmentations along the length of the vessel. A variety of techniques are available in SimVascular
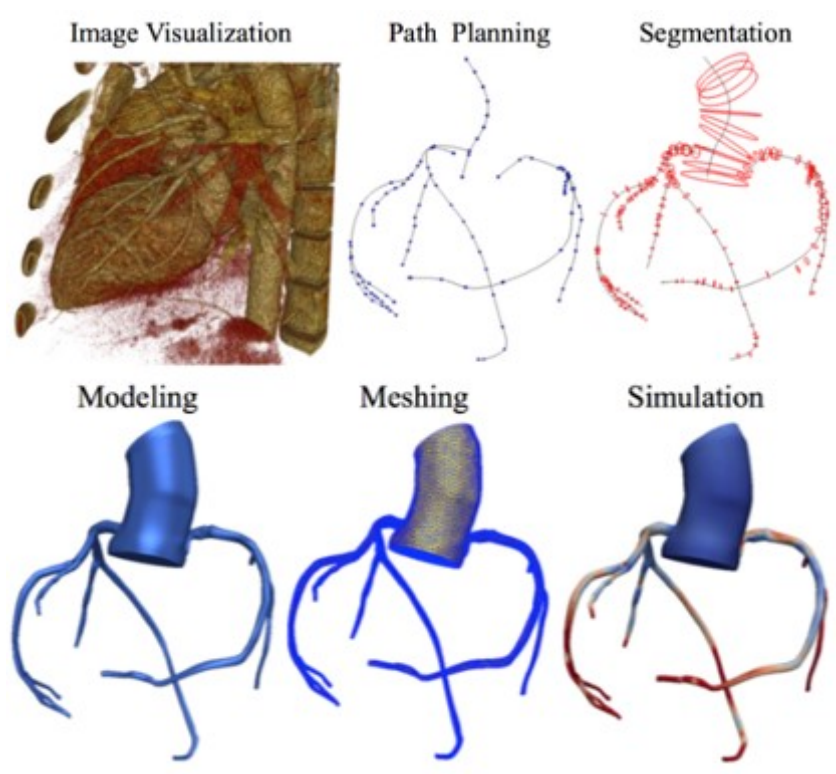

Figure 1: The SimVascular pipeline begins with volumetric medical imaging data and results in blood flow simulation based on six common steps as shown [3].

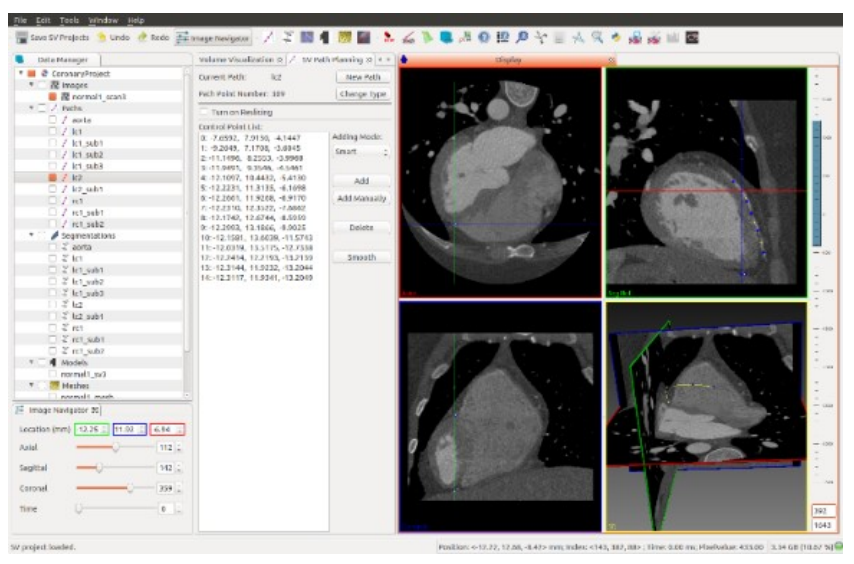

Figure 2: The SimVascular Graphical User Interface [3].

including thresholding and level-set based methods. Recent work has highlighted the potential for machine learning to accelerate this process. Once the segmentations are created, a 3-D tubular vessel volume is lofted from each set of ordered image segmentations. This can either be done using CAD algorithms (e.g. OpenCASCADE, www.opencascade.org) or integrated techniques to generate high quality triangulated surfaces. After 3-D solids exist for each vessel, Boolean operations are performed to create a single volume representing the flow domain. Geometric based surface smoothing may optionally be applied at this point to smooth the junctions of vessel branches. 


\subsection{Preparing \& Running Analyses}

After the anatomic model is complete, boundary surfaces can be tagged as needed. A tetrahedral mesh is generated for the fluid domain and nodal connectivity, element connectivity, and surface information is written to files to be used by the blood flow solver. Depending on the anatomic region and the analysis to be performed, boundary conditions may be tuned to match clinically available data or published population based data. Simulations are typically carried out either on a desktop computer or a HPC depending on the computational requirements for the simulation. In a standard workflow, an analyst may perform multiple steady and pulsatile simulations on a very coarse mesh for determining appropriate outlet boundary conditions. An adapted or refined mesh is then typically run on an HPC machine such as COMET. While there is significant variation throughout different computational studies, a "rule of thumb" is that an analysis on a multi-million element mesh will typically take about 24-48 hours on 32 cores for five or six cardiac cycles of simulation. Since the initial conditions of the flow domain prior to the simulation are unknown for a living patient, transient features exist that may dissipate under laminar or transitional flow conditions such that the analyst often only post-processes the final cardiac cycle or two of the simulation.

\subsection{Visualizing Results}

After the simulation has completed, the results are retrieved (if necessary) from the HPC cluster, post-processed, and visualized on the analyst's desktop or laptop. Flow features such as pressure, velocity, volumetric flow rates, wall shear stress, oscillating shear index, etc. may be calculated or visualized using packages such as ParaView (www.paraview.org). A quality research simulation can involve several gigabytes (or much more) of simulation results files to be processed.

\section{SIMVASCULAR GATEWAY}

\subsection{Overview}

As discussed above, the SimVascular workflow consists of both interactive steps best done on a local desktop and computing-intensive steps best done on remote HPCs, preferably using a version of the SimVascular software installed and maintained by the SimVascular development team. We have developed the SimVascular Gateway (SVG) to provide access to the solver on HPC resources. SVG is designed to support three core user groups: students enrolled in cardiovascular modeling courses, researchers otherwise without access to HPC, active research labs with XSEDE allocations and graduate students unfamiliar with HPC administration and/or summer interns and undergraduate researchers.

These groups have some differences, but many common needs as well. A primary difference between experienced users and novices is the ability to verify input files prior to submission to reduce wasted compute cycles, insight into balancing mesh density and time steps to maximize quality of the results while reducing computational needs, and general understanding of the shared nature of HPC resources. For example, our collective experience has been that many first-time graduate students or course participants will submit every job, regardless of size, to the maximize allowable cores on the
HPC. Designing the SVG to be useful for both advanced users and novice users requires policy enforcement to reduce computational waste as discussed below.

\subsection{Access \& Managing the Gateway}

The potential users span numerous campuses and research laboratories across the country. User authentication, password security, and user administration for this group can be complex. The SVG relies primarily on CILogon (www.cilogon.org) [2] for user authentication. As all of our users have active affiliations with universities, most of which support CILogon, this provides a secure and robust authentication method for the SVG. While CILogon generally works well, our early experience has shown that some schools have unusual implementations of CILogon preventing users from accessing the SVG. For these users or other users without CILogon access, local accounts can be generated and administered by an admin [5]. SVG users have the ability to browse projects and browse experiments, while administrators additionally have the ability to display experiment statistics and to manage users access.

\subsection{Groups \& Classes on the Gateway}

A feature under development will enable the SVG to have configurable user groups with different permission levels (e.g. restrictions on node count, total core count, and wall clock time). This will enable a given professor to configure both a "research" user with more access to resources and restrict students in a course from running larger simulations. In addition, instructors may need access to students' work for hands-on student projects, and gateway resources to facilitate adoption of these tools for education. To share an allocation with students, a professor would first create a group for the class in the SVG (see Fig. 3). The professor can give the group a name and description and assign students to the group. In addition, the professor can designate certain other SVG users as admins of the group. These group admins can also edit the list of members of the group. This allows the professor to delegate the task of adding students to the classroom group to a teaching assistant (TA). Second, a professor would then create a description of one or more compute resources and the allocation number to use when submitting jobs to those resources. In Airavata this description of shared resources is called a "Group Resource Profile" since it is a profile of resources that are shared with a group (see Fig. 3). In addition to listing the compute resources and allocation to charge, the professor can specify in the Group Resource Profile the compute cluster queues that can be used. For each allowed queue the professor can further restrict the maximum allowed CPU core count, the maximum allowed node count and the maximum allowed wall clock time limit for each job. Finally, a professor would pick the classroom group, previously created, that would have access to this Group Resource Profile. Now any member of the classroom group can make use of the computational resources defined in the Group Resource Profile.

\subsection{Running Computational Experiments on the Gateway}

The SVG enables the user to create computational experiments. When an SVG user runs SimVascular they may specify a name for 
the experiment, select a project in which to store the computational experiment, and upload the input files required by the SimVascular application. Now an SVG classroom user will also choose a Group Resource Profile that has been shared with them from a drop down UI element (labeled here as "Allocation"). The classroom user will be able to change the core count, node count, and max wall clock time but only within the limits specified by the professor for the selected Group Resource Profile.

\subsection{Initial Experience with the Gateway for Education}

We have deployed the SVG for use in hands-on class projects in courses on computational modeling in the cardiovascular system, at Purdue University and Marquette University since the Gateway launch in fall 2017. Students in all classes participated remotely in tutorials, online help sessions, and demos run by the SV development team. Each student was given a set of medical image data, and was asked to build an anatomic model, generate a mesh, assign boundary conditions, run a simulation on the Gateway and analyze the results. Students reported (in oral presentations and written reports) on their disease topic and clinical relevance, methods chosen for simulations, including mesh convergence and boundary condition choices, and analysis of at least two relevant flow quantities (e.g. wall shear stress). This provided students with hands on interactive experiences in a way that was accessible to students from a range of backgrounds. Formal assessments on the use of SimVascular as an educational tool were previously reported by our team [1]. The Gateway also provided students a user-friendly initiation into HPC computing and access to resources not otherwise available to them.

\section{CONCLUSIONS}

SimVascular has been successfully integrated into graduate level courses on cardiovascular modeling at multiple schools across the country to introduce state-of-the-art modeling to the students and provide hands-on experience with blood flow simulation and HPC. While the front-end application can be installed and run locally on a laptop, the flow solver requires HPC, which is typically not available for educational purposes due to limitations in both resources and expertise. Here, we describe an Apache Airavata-based science gateway to address these needs. XSEDE's Comet provides the backend computing power. Our approach allows the SimVascular team to provision HPC resources and install and maintain the software. The science gateway interface provides access to SimVascular's flow solver, while allowing students to use SimVascular's desktop interfaces on their local machine. Our initial experience deploying the Gateway in classes was favorable, and provides students a user friendly initiation to blood flow simulation in an HPC environment.

\section{ACKNOWLEDGMENTS}

This work was partially supported by funding from the NSF (Awards 1339824 and 1663671) and the National Institute of Biomedical Imaging and Bioengineering (1R01EB018302-01A1). XSEDE is funded by NSF Award 1548562. Apache Airavata and SciGaP services are supported by NSF Award 1339774.

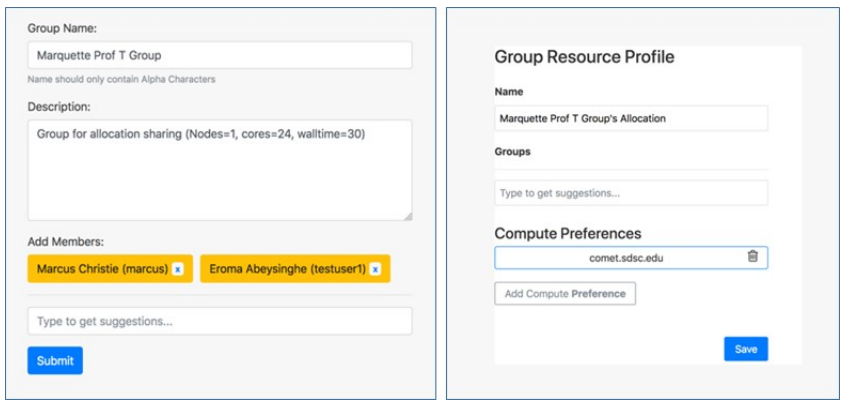

Figure 3: Creating a classroom group (left). Group Resource Profile for sharing an allocation with a classroom (right).

\section{REFERENCES}

[1] Craig J. Goergen, Shawn C. Shadden, and Alison L. Marsden. 2017. SimVascular as an instructional tool in the classroom. In 2017 IEEE Frontiers in Education Conference (FIE). 1-4. https://doi.org/10.1109/FIE.2017.8190438

[2] Basney Jim, Fleury Terry, and Gaynor Jeff. [n. d.]. CILogon: A federated X.509 certification authority for cyberinfrastructure logon. Concurrency and Computation: Practice and Experience 26, 13 ([n. d.]), 2225-2239. https://doi.org/10.1002/cpe.3265

[3] Hongzhi Lan, Adam Updegrove, Nathan M Wilson, Gabriel D. Maher, Shawn Shadden, and Alison Marsden. 2018. A Re-Engineered Software Interface and Workflow for the Open-Source SimVascular Cardiovascular Modeling Package. fournal of Biomechanical Engineering 140, 2 (2018). https://doi.org/10.1115/1. 4038751

[4] Suresh Marru, Lahiru Gunathilake, Chathura Herath, Patanachai Tangchaisin, Marlon Pierce, Chris Mattmann, Raminder Singh, Thilina Gunarathne, Eran Chinthaka, Ross Gardler, Aleksander Slominski, Ate Douma, Srinath Perera, and Sanjiva Weerawarana. 2011. Apache Airavata: A Framework for Distributed Applications and Computational Workflows. In Proceedings of the 2011 ACM Workshop on Gateway Computing Environments (GCE '11). ACM, New York, NY, USA, 21-28. https://doi.org/10.1145/2110486.2110490

[5] Supun Nakandala, Hansini Gunasinghe, Suresh Marru, and Marlon Pierce. 2016. Apache Airavata security manager: Authentication and authorization implementations for a multi-tenant escience framework. In 2016 IEEE 12th International Conference on e-Science (e-Science). 287-292. https://doi.org/10.1109/eScience.2016. 7870911

[6] John Towns, Timothy Cockerill, Maytal Dahan, Ian Foster, Kelly Gaither, Andrew Grimshaw, Victor Hazlewood, Scott Lathrop, Dave Lifka, Gregory D. Peterson, Ralph Roskies, J. Ray Scott, and Nancy Wilkins-Diehr. 2014. XSEDE: Accelerating Scientific Discovery. Computing in Science Engineering 16, 5 (Sept 2014), 62-74. https://doi.org/10.1109/MCSE.2014.80

[7] Nancy Wilkins-Diehr, Sergiu Sanielevici, Jay Alameda, John Cazes, Lonnie Crosby, Marlon Pierce, and Ralph Roskies. 2016. An Overview of the XSEDE Extended Collaborative Support Program. In High Performance Computer Applications, Isidoro Gitler and Jaime Klapp (Eds.). Springer International Publishing, Cham, 3-13. 